\title{
EXPLOSIONS IN COMPLETELY UNSTABLE FLOWS. I PREVENTING EXPLOSIONS
}

BY

\author{
ZBIGNIEW NITECKI
}

\begin{abstract}
Several conditions are equivalent to the property that a flow (on an open manifold) and its $C^{0}$ perturbations have only wandering points. These conditions are: (i) there exists a strong Liapunov function; (ii) there are no generalized recurrent points in the sense of Auslander; (iii) there are no chain recurrent points, in the sense of Conley; (iv) there exists a fine sequence of filtrations; (v) relative to some metric; the flow is the gradient flow of a function without critical points. We establish these equivalences, and consider a few questions related to structural stability when all orbits wander.
\end{abstract}

For dynamical systems on a compact manifold, the phenomenon of " $\Omega$-explosions" is well understood. Several sets of necessary and sufficient conditions have been formulated ([13], [11]) for the nonwandering set, $\Omega$, regarded as a set-valued map on the space of diffeomorphisms or flows on a compact manifold, to be upper semi-continuous (in the $C^{0}$ topology) at a given point.

In this paper, we study the analogous phenomenon for a flow on an open manifold with all orbits wandering. We give several characterizations of the $\left(C^{0}\right)$ interior of the set of flows with $\Omega=\varnothing$. Definitions will be given in the text of the paper, but the results are summarized in the following

THEOREM. Suppose $\varphi$ is a flow on the open manifold $M$, with velocity vectorfield $\dot{\varphi}=X$, and $\Omega(\varphi)=\varnothing$. Then the following conditions are equivalent:

1. $\varphi$ is nonexplosive.

2. There exists a strong Liapunov function for $\varphi$.

3. The Auslander recurrent set of $\varphi$ is empty.

4. The Conley recurrent set of $\varphi$ is empty.

5. There exists a fine sequence of filtrations for $\varphi$.

6. $X$ is the gradient vectorfield (with respect to some metric) of a function without critical points on $M$.

This theorem was announced in the note [9]. After [9] was written, the paper of Takens and White [14] appeared, in which the equivalence $2 \Leftrightarrow 3$

Received by the editors March 10, 1977 and, in revised form, May 4, 1977.

AMS (MOS) subject classifications (1970). Primary 58F10; Secondary 34C35, 54H20.

$K e y$ words and phrases. Completely unstable flow, $\Omega$-explosion, chain recurrence, generalized recurrence, prolongation, gradient flow, Liapunov function, filtration, structural stability.

(C) American Mathematical Society 1979 
(originally due to Auslander [1], [2]) and the implication $2 \Rightarrow 1$ are noted, while the implication $1 \Rightarrow 2$ is conjectured. Takens and White also prove the genericity, for vectorfields in the strong $C^{r}$ topology (any $r$ ), of a condition that implies 2 when $\Omega=\varnothing$. On the other hand, it is shown in [10] that, with two exceptions, every open manifold supports flows for which condition 2 fails, and hence $\Omega(\varphi)=\varnothing$ but $\Omega(\psi) \neq \varnothing$ for certain $C^{0}$ perturbations of $\varphi$.

This paper is divided into sections focused on each of the six conditions above. $\$ 1$ contains the basic definitions related to explosions. $\$ 2$ discusses Liapunov functions, and establishes the implication $2 \Rightarrow 1$ (Proposition 1). $\$ 3$ recalls the work of Auslander on "generalized recurrence", which shows $2 \Leftrightarrow 3$ (Proposition 2). $\$ 4$ reformulates, in the context of open manifolds, the work of Conley on "chain recurrence", and establishes $4 \Rightarrow 3$ (Lemma 5) and $1 \Rightarrow 4$ (Proposition 4). This completes the cycle $4 \Rightarrow 3 \Rightarrow 2 \Rightarrow 1 \Rightarrow 4$, and so in particular proves the conjecture of Takens and White (at least for $C^{0}$ perturbations). $\$ 5$ reformulates the various notions of filtration from [11] and [13], and establishes the equivalence $1 \Leftrightarrow 5$ (Proposition 5) by means of $2 \Rightarrow 5 \Rightarrow 4$ (Lemmas 6 and 7). In passing, it is noted that even when $\Omega=\varnothing$. the existence of a fine sequence of filtrations does not imply existence of a fine filtration (Lemma 8 and corollary). $\$ 6$ establishes the equivalence $2 \Leftrightarrow 6$; in fact, the functions in conditions 2 and 6 can be taken to be the same.

Finally, in \$7, we recall the structural stability results of Krych [6] and Mendes [7]. The formulation of their results in our special case-that is, for a completely unstable flow-turns out to be the relatively easy fact that a parallelizable flow is structurally stable. We close with an easy example of a completely unstable flow which is nonexplosive (hence $\Omega$-stable) but not structurally stable. A more difficult example of Takens and White shows that a whole open set of completely unstable flows in the plane are not structurally stable.

1. Basic definitions. We begin with a brief review of some terminology and notation. By an open manifold, we mean a connected, finite-dimensional, paracompact, $C^{\infty}$ manifold (without boundary), which is not compact. A flow $\varphi: R \times M \rightarrow M$ will be denoted in one of two forms: $\varphi(t, x)=\varphi^{t}(x)$. Given a flow $\varphi$, we call an open set $U \subset M$ wandering for $\varphi$ if there exists $T \in R$ such that $\varphi^{t}(U) \cap U=\varnothing$ for $|t| \geqslant T$. We will call $U$ unrevisited if it is wandering and the intersection of any $\varphi$-orbit with $U$ is connected-that is, if $x \in U$ and $\varphi^{t}(x) \in U$, then $\varphi^{s}(x) \in U$ for all $s, 0<s<t$. Using the local parallelizability of a flow near a nonequilibrium point, it is easy to see that every point of a wandering open set $U$ has an unrevisited neighborhood contained in $U$.

A point $x \in M$ is nonwandering under $\varphi$ if it has no wandering neighborhood. The (closed, $\varphi$-invariant) set of all nonwandering points for $\varphi$ is 
denoted $\Omega(\varphi)$. A flow for which $\Omega(\varphi)=\varnothing$ is called completely unstable. This use of the word "unstable" may occasion some confusion when speaking of structural stability (a "completely unstable" flow can be "structurally stable"). However, this terminology has been in wide use for a long time [8], and we shall not venture into revisionism on this point.

When we speak of perturbations of a flow, we will refer to the strong (Whitney) topology. Our main concern will be with $C^{0}$ perturbations: given a Riemann metric on $M$, a $C^{0}$-neighborhood of a vectorfield $X$ on $M$ will be specified by a positive function $\varepsilon(x)$ on $M$, and will consist of all vectorfields $Y$ satisfying the pointwise estimate

$$
\|X(x)-Y(x)\|<\varepsilon(x) \text { for all } x \in M .
$$

The flows on $M$ will be topologized by means of their velocity vectorfields. Thus, a $C^{0}$-neighborhood of the $C^{1}$ flow $\varphi$ will consist of the $C^{1}$ flows $\psi$ for which

$$
\|\dot{\varphi}(x)-\dot{\psi}(x)\|<\varepsilon(x) \text { for all } x \in M .
$$

Although we are invoking a metric on $M$ to define it, the strong $C^{0}$ topology is independent of the metric we use.

There are, of course, some technical problems concerning the local structure of the space of flows with this topology-specifically, a vectorfield $Y$ which is near $X=\dot{\varphi}$ may fail to generate a flow, either because the differential equation

$$
d x / d t=Y(x)
$$

may have nonunique solutions (if $Y$ is not Lipschitz), or because the solutions are not defined for all time ( $Y$ may not be "complete"). However, all actual perturbations which we construct in this paper will be given by flows whose velocity agrees with the unperturbed velocity off a compact set; and all general results will use only the existence of a velocity vectorfield $Y=\dot{\psi}$ $C^{0}$-near $X=\dot{\varphi}$. Thus, these technicalities need not trouble us in considering the problem of $\Omega$-explosions.

The problem concerning us in this paper can be stated thus: suppose the flow $\varphi$ is completely unstable; what conditions will guarantee that every flow $\psi$ in some $C^{0}$-neighborhood of $\varphi$ is also completely unstable? When a flow with $\Omega(\varphi)=\varnothing$ can be perturbed so that $\Omega(\psi) \neq \varnothing$, then we have the analogue of a phenomenon ([12], [13], [11]) known as an " $\Omega$-explosion". We will call a flow $\varphi$ explosive if $\Omega(\varphi)=\varnothing$ but every $C^{0}$-neighborhood of $\varphi$ contains flows $\psi$ with $\Omega(\psi) \neq \varnothing$.

There is a standard example, due to $\mathrm{J}$. Palis [12], of a diffeomorphism of a plane region whose nonwandering set consists of six fixed points, but such that by perturbing $f$ on two discs far from these fixed points, one obtains an infinite nonwandering set which intersects these discs. Papers that have 
considered various aspects of structural stability on open manifolds ([7], [6], [14]) have all noted that examples of explosive diffeomorphisms can be obtained by deleting the six fixed points from the plane in Palis' example. We discuss a large class of explosive flows in a related paper [10]; in particular, they exist on all open manifolds except the line and the plane.

2. Explosions and Liapunov functions. In [7], Mendes showed that a completely unstable diffeomorphism of the plane is not explosive; using the Brouwer translation theorem, he constructed a continuous function which was decreasing along orbits. We review here the implications for explosiveness of the existence of such a function. The same results have been noted recently by Takens and White [14].

DEFINITION. Given a flow $\varphi$ on $M$,

(i) a Liapunov function for $\varphi$ is a continuous real-valued function $f: M \rightarrow R$ such that $f\left(\varphi^{t}(x)\right) \leqslant f(x)$ for all $x \in M$ and all $t \geqslant 0$;

(ii) a function as in (i) is a strict Liapunov function for $\varphi$ if the inequality above is strict: $f\left(\varphi^{t}(x)\right)<f(x)$ for all $x \in M$ and $t>0$.

We note the following fact, which can be established by convolution methods of Wilson [15].

Lemma 1. If $\varphi$ possesses a continuous (strict) Liapunov function, then it possesses a $C^{\infty}$ (strict) Liapunov function.

Thus, we can assume $f$ in the above definition is smooth. In this case, the definition can be reformulated, in a somewhat strengthened form. Wilson's results lets us assume that the strengthened form below holds. Let $X=\dot{\varphi}$.

(i) $f$ is Liapunov iff $X f(x)(=d f(X)) \leqslant 0$ for all $x \in M$.

(ii) $f$ is strictly Liapunov if $X f(x)<0$ for all $x \in M$.

For convenience, we shall refer to a function satisfying condition (ii) as a strong Liapunov function.

Mendes' method of showing nonexplosiveness can be summarized in two observations:

LEMMA 2. If $X f(x)<0$ for all $x \in M$, then there exists a $C^{0}$-neighborhood $\mathfrak{U}$ of $X$ such that $Y f(x)<0$ for all $x \in M$ and all $Y \in \mathfrak{U}$.

Proof. Immediate; this is a pointwise condition, which could be written, in terms of a metric, as $Y \cdot \operatorname{grad} f<0$.

Lemma 3. If $\varphi$ possesses a strict Liapunov function, then $\varphi$ is completely unstable.

Proof. Pick a compact neighborhood $U$ of $x \in M$ such that

$$
f\left(\varphi^{1}(x)\right)<\min \{f(y) \mid y \in U\} .
$$


By continuity of $\varphi^{1}$, there exists a smaller neighborhood $V \subset U$ of $x$ such that

$$
\max f\left|\varphi^{1}(V)<\min f\right| U \leqslant \inf f \mid V .
$$

Thus, $V$ is an unrevisited neighborhood of $x$.

We have, then,

Proposition 1. If $\varphi$ possesses a strict Liapunov function, then it is nonexplosive.

Proof. By [15], there exists $f$ with $X f<0$, where $X=\dot{\varphi}$. By Lemma 2, this condition persists under perturbation, and, by Lemma 3 , implies complete instability for a $C^{0}$-neighborhood of $\varphi$.

3. The Auslander recurrent set. As a consequence of the theory developed by J. Auslander and P. Seibert ([1], [2], or see [3, Chapter 7]), the existence of strict Liapunov functions can be characterized in terms of prolongations. We review the necessary definitions here.

Definition. Given $x \in M$ and a flow $\varphi$ on $M$, define the first prolongational limit set of $x$ by

$$
\begin{aligned}
J_{1}^{+}[x] & =\left\{y \mid \text { there exist } x_{n} \rightarrow x, t_{n} \rightarrow+\infty \text { such that } \varphi\left(t_{n}, x_{n}\right) \rightarrow y\right\} \\
& =\bigcap\{\omega(U) \mid U \text { is a neighborhood of } x\} .
\end{aligned}
$$

Here, $\omega(U)=\bigcap_{T>0} \operatorname{clos} \cup_{t>T} \varphi^{t}(U)$.

We need to define higher prolongations; to this end, we define two operations on set-valued maps. Suppose $S: M \rightarrow 2^{M}$ is a map assigning to each point $x \in M$ a subset $S(x) \subset M$. We extend $S$ to subsets $A \subset M$ by $S(A)=\cup\{S(x) \mid x \in A\}$ and define two new set-valued maps by

(i) $\delta S(A)=S(A) \cup S(S(A)) \cup \ldots$,

(ii) $\mathscr{D} S(x)=\cap\{\operatorname{clos} S(U) \mid U$ a neighborhood of $x\}$ and $\mathscr{D} S(A)=U$ $\{\mathscr{D} S(x) \mid x \in A\}$.

Then the first prolongational limit could be defined as

$$
J_{1}^{+}[x]=(\mathscr{D} \omega)(x) \text {. }
$$

We define a higher prolongation for each ordinal, $\alpha$, by transfinite induction:

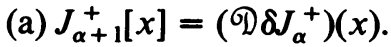

(b) If $\alpha$ is a limit ordinal,

$$
J_{\alpha}^{+}[x]=\mathscr{D}\left[\cup\left\{\delta J_{\beta}^{+}[x] \mid \beta<\alpha\right\}\right] .
$$

Finally, we define the following notion, called "generalized recurrence" by J. Auslander [1].

DEFINITION. The Auslander recurrent set of a flow $\varphi$ is

$$
R_{A}(\varphi)=\left\{x \in M \mid x \in J_{\alpha}^{+}[x] \text { for some ordinal } \alpha\right\} .
$$


It is clear from the definitions that, in general (on a locally compact space), $\Omega(\varphi) \subset R_{A}(\varphi)$-in fact, the definition of $\Omega$ could be reformulated as

$$
\Omega(\varphi)=\left\{x \mid x \in J_{1}^{+}(x)\right\} .
$$

The main interest in the Auslander set stems from the following theorems; for their proof, we refer the reader to [1] or [3].

Proposition 2 (Auslander). 1. A point $x \in M$ belongs to $R_{A}(\varphi)$ if and only if every Liapunov function for $\varphi$ is constant along the orbit of $x$.

2. There exists a Liapunov function for $\varphi$ which, on the complement of $R_{A}(\varphi)$, is strict Liapunov.

Thus, the existence of a strict Liapunov function for $\varphi$ is equivalent to the condition that $R_{A}(\varphi)$ be empty. Combining with Proposition 1, this gives

Proposition 3. $R_{A}(\varphi)=\varnothing$ implies $\varphi$ is nonexplosive.

4. The Conley recurrent set. Another notion of recurrence was formulated by C. Conley [4] for dynamical systems on compact spaces. The definitions below seem to form natural extensions of Conley's ideas to the case of open manifolds.

Definition. Fix a flow, $\varphi$, and a Riemann metric on $M$, with geodesic distance $d$. Given a function $\varepsilon(x)>0$ on $M$ and a real number $T>0$,

1. An $(\varepsilon, T)$-chain for $\varphi$ is a (finite or countable) sequence of points $x_{n} \in M$ and times $t_{n} \geqslant T$ such that

$$
d\left[\varphi\left(t_{n}, x_{n}\right), x_{n+1}\right]<\varepsilon\left(\varphi\left(t_{n}, x_{n}\right)\right) .
$$

2. The points which are $(\varepsilon, T)$-accessible from $x$ (under $\varphi$ ) are

$$
P(\varepsilon, T, x)=\left\{y \mid y=x_{k} \text { for some finite }(\varepsilon, T) \text {-chain with } x_{0}=x\right\} \text {. }
$$

The following is called the "chain recurrent set" by Conley.

Definition. The Conley recurrent set of a flow is

$R_{C}(\varphi)=\{x \mid x \in P(\varepsilon, T, x)$ for all functions $\varepsilon(x)>0$ and all constants $T>0\}$.

For our purposes in dealing with flows, it is useful to consider the following variation on the notion of an $(\varepsilon, T)$-chain.

Definition. Fix a flow $\varphi$ and a Riemann metric, \|\| , on $M$. Given a function $\varepsilon(x)>0$, an $\varepsilon$-quasiorbit for $\varphi$ is a parametrized curve $\gamma: R \rightarrow M$ such that

$$
\|\dot{\gamma}(t)-X(\gamma(t))\|<\varepsilon(\gamma(t)) \text { for all } t .
$$

Here, as usual, $X=\dot{\varphi}$ is the velocity vectorfield of $\varphi$; we note that a quasiorbit, in contrast to a true trajectory, is allowed to cross itself.

We can then reformulate the Conley definition by the following:

LEMMA 4. $x \in R_{C}(\varphi)$ if and only if, for every positive function $\varepsilon: M \rightarrow R$, 
there exists an $\varepsilon$-quasiorbit of $\varphi$ which hits $x$ twice.

Proof. Note that an $\varepsilon$-quasiorbit hitting $x$ twice can always be replaced by an $\varepsilon$-quasiorbit which is periodic (i.e., where $\gamma$ is really a map of the circle into $M$ ). By the basic estimates giving continuous dependence of solutions on initial conditions for ordinary differential equations, given a function $\varepsilon(x)>$ 0 and $T>0$ there exists a function $\delta(x)>0$ such that for any $\delta$-quasiorbit $\gamma$ the points $x_{n}=\gamma\left(t_{n}\right)$, where $t_{0}=0$ and $t_{n-1}+T \leqslant t_{n} \leqslant t_{n-1}+2 T$, form an $(\varepsilon, T)$-chain for $\varphi$.

Conversely, given a positive function $\varepsilon(x)$, and a large positive constant $T$, there exists a (small) positive function $\delta(x)$ so that any point $y$ with $d\left(\varphi^{t}(x), y\right)<\delta\left(\varphi^{t}(x)\right)$ for some $t>T$ can be reached by an $\varepsilon$-quasiorbit starting at $x$.

Thus, accessibility under all chains is equivalent to accessibility under quasiorbits, and the lemma is proven.

The notion of accessibility by quasiorbits is related to that of "accessibility under constantly-acting disturbances." Auslander and Seibert [2] prove a result in this context which implies the inclusion $R_{A} \subset R_{C}$. This inclusion is proven explicitly by Conley [4] when $M$ is compact and the functions $\varepsilon(x)$ in the above definitions are replaced by constants. Since we have extended Conley's definition to fit our context, we show how his pattern of proof can be followed to prove the corresponding inclusion here.

LeMMA 5. $R_{A}(\varphi) \subset R_{C}(\varphi)$.

Proof. We begin by defining the "chain-accessible" set of a point under a given flow:

$$
P^{+}(x)=\bigcap\{P(\varepsilon, T, x) \mid T>0 \text { and } \varepsilon: M \rightarrow R \text { is positive }\} .
$$

Note that $P^{+}(x)$ is closed, for we could replace the strict inequality in the definition of $P(\varepsilon, T, x)$ with weak inequality, and thus express $P^{+}$as an intersection of closed sets.

Thus, $R_{C}(\varphi)=\left\{x \mid x \in P^{+}(x)\right\}$ and

$$
R_{A}(\varphi)=\left\{x \mid x \in J_{\alpha}^{+}[x] \text { for some ordinal } \alpha\right\} .
$$

It suffices to show that, for any ordinal $\alpha, J_{\alpha}^{+}[x] \subset P^{+}(x)$. We prove this by transfinite induction.

Step 1. $J_{1}^{+}[x] \subset P^{+}(x)$. Given $x_{n} \rightarrow x, t_{n} \rightarrow+\infty, y=\lim \varphi\left(t_{n}, x_{n}\right)$, and the function $\varepsilon(x)>0$, we must find an $\varepsilon$-quasiorbit joining $x$ to $y$. Pick $N_{1}$ so that, for each $n>N_{1}$,

(i) $t_{n}>T$,

(ii) there exists an $\varepsilon$-quasiorbit joining $x$ to $\varphi\left(T, x_{n}\right)$.

This is, of course, possible because for $T$ fixed, $\varphi(T, x)=\lim \varphi\left(T, x_{n}\right)$. Now, 
pick $N_{2}>N_{1}$ so that, for $n>N_{2}$,

(iii) $t_{n}>2 T$,

(iv) there exists an $\varepsilon$-quasiorbit joining $\varphi\left(t_{n}-T, x_{n}\right)$ to $y$.

This is possible because $\varphi(-T, y)=\lim \varphi\left(t_{n}-T, x_{n}\right)$. Finally, pick any $n>N_{2}$. Form the curve $\gamma(t)$, by following the $\varepsilon$-quasiorbit of (ii) from $x$ to $\varphi\left(T, x_{n}\right)$, then the orbit of $\varphi$ from $\varphi\left(T, x_{n}\right)$ to $\varphi\left(t_{n}-T, x_{n}\right)$, and finally the $\varepsilon$-quasiorbit in (iv) from $\varphi\left(t_{n}-T, x_{n}\right)$ to $y$. Then $\gamma$ satisfies the conditions for an $\varepsilon$-quasiorbit except that it may have corners at the two points $\varphi\left(T, x_{n}\right)$ and $\varphi\left(t_{n}-T, x_{n}\right)$. But at these points, both one-sided derivatives exist and satisfy the required inequalities; thus it is possible to smooth $\gamma$ near these points without violating the inequalities, to obtain an $\varepsilon$-quasiorbit from $x$ to $y$.

Step 2. $J_{\alpha}^{+}[x] \subset P^{+}(x)$ for all $\alpha$. To prove an induction step, we need to show two inclusions:

(a) $\delta P^{+}(A) \subset P^{+}(A)$ for all subsets $A \subset M$.

(b) $\mathscr{D} P^{+}(A) \subset P^{+}(A)$ for all subsets $A \subset M$.

(a) To establish (a), note that for any point $x \in M$,

$$
P^{+}\left(P^{+}(x)\right)=P^{+}(x)
$$

because $\varepsilon$-quasiorbits of sufficient length can be pieced together and smoothed (much like what we did in Step 1). Since this is true for all points $x \in M$, it is true for subsets, and $\delta P^{+}(A)=P^{+}(A)$ for $A \subset M$.

(b) Now, by continuous dependence on initial conditions, if a sufficiently long $\varepsilon$-quasiorbit comes sufficiently close to a given point $x \in M$, then its initial segment can be replaced by a segment starting from $x$ without violating the estimates in the definition of quasiorbit. In other words, for a sufficiently small neighborhood $U$ of $x$ and sufficiently large $T$, any point accessible at time $T$ by an $\varepsilon$-quasiorbit starting in $U$ can be reached by an $\varepsilon$-quasiorbit starting at $x$; thus

$$
P^{+}(U)=P^{+}(x) \text {. }
$$

But $P^{+}(x)$ is closed, so this implies that for all points $x \in M$,

$$
\mathscr{D} \delta P^{+}(x)=\mathscr{D} P^{+}(x)=P^{+}(x) \text {. }
$$

Now, we know that $J_{1}^{+}[x] \subset P^{+}(x)$, and that, if $J_{\alpha}^{+}[x] \subset P^{+}(x)$, then

$$
J_{\alpha+1}^{+}[x]=\mathscr{D} \delta J_{\alpha}^{+}[x] \subset \mathscr{D} \delta P^{+}(x)=P^{+}(x) \text {. }
$$

Since this is true for every point $x \in M$, it is also true for subsets. If $\alpha$ is a limit ordinal and $J_{\beta}^{+}[x] \subset P^{+}(x)$ for every $\beta<\alpha$, then

$$
\begin{aligned}
J_{\alpha}^{+}[x] & =\mathscr{D} \cup\left\{\delta J_{\beta}^{+}(x) \mid \beta<\alpha\right\} \subset \mathscr{D} \cup\left\{\delta P^{+}(x)\right\} \\
& =\mathscr{D} \cup P^{+}(x)=\mathscr{D} P^{+}(x)=P^{+}(x),
\end{aligned}
$$

and our lemma has been proven, by transfinite induction: if $x \in R_{A}(\varphi)$, then $x \in J_{\alpha}^{+}[x] \subset P^{+}(x)$, and so $x \in R_{C}(\varphi)$. Q.E.D. 
The content of this argument is that every point in a higher prolongational limit set can be reached by a quasiorbit.

In general, the Conley set $R_{C}$ is strictly larger than the Auslander set, $R_{A}$. For example, the flow in the plane defined by

$$
\dot{x}=x^{2}+y^{2}-1, \quad \dot{y}=0,
$$

has

$$
R_{A}=\left\{(x, y) \mid x^{2}+y^{2}=1\right\}
$$

while

$$
R_{C}=\left\{(x, y) \mid x^{2}+y^{2} \leqslant 1\right\} .
$$

However, when the Auslander set is empty, the Conley set is empty as well. While this fact will be a by-product of our main result, it can be established somewhat more directly as a consequence of Proposition 2: for if $R_{A}=\varnothing$, then there exists a strong Liapunov function $f$ for $\varphi$, and by Lemma 2, for any sufficiently small positive function $\varepsilon, f$ must also decrease strictly along any $\varepsilon$-quasiorbit. In particular, there are no closed quasiorbits, and so $R_{C}=\varnothing$.

Finally, we can use an idea from [11] to show that if $R_{C} \neq \varnothing$, then $\varphi$ is explosive-that is, to show that condition 1 implies condition 4 in our main theorem:

Proposition 4. Suppose $\Omega(\varphi)=\varnothing$. If $R_{C}(\varphi) \neq \varnothing$, then $\varphi$ is explosive.

Proof. We invoke the characterization of $R_{C}$ in terms of quasiorbits in Lemma 4. Given an $\varepsilon(x)$-neighborhood of $\varphi$, suppose there exists a closed $\delta(x)$-quasiorbit for $\varphi$, passing through $x$, where $0<\delta(x)<\varepsilon(x)$. The closed quasiorbit can be replaced by a $C^{\infty}$ simple, closed curve $\gamma$ which is still a $\delta(x)$-quasiorbit for $\varphi$ : in dimension 3 or more, this can be accomplished by transversality arguments, while in dimension 2, we throw away all "loops" except one containing $x$, then smooth as before at corners.

Now, picking a thin tubular neighborhood $U$ about the curve $\gamma$, we can extend the $C^{\infty}$ vectorfield $\dot{\gamma}$ defined along $\gamma$ to a $C^{\infty}$ vectorfield $Y$ defined on $U$ for which

$$
\|X(x)-Y(x)\|<\varepsilon(x), \quad x \in U .
$$

We then pick a smooth function $\lambda: M \rightarrow[0,1]$ with

$$
\lambda(\gamma(t))=1, \quad \lambda(x)=0 \quad \text { for } x \notin U
$$

and define a new vectorfield by

$$
Z(x)=\lambda(x) Y(x)+[1-\lambda(x)] X(x) .
$$

This vectorfield agrees with $X$ off $U$, is as smooth as $X$, and so defines a flow $\psi$ on $M$; furthermore, 


$$
\|Z(x)-X(x)\| \leqslant|\lambda(x)|\|Y(x)-X(x)\|<\varepsilon(x)
$$

and so $\psi$ is a flow in the $\varepsilon(x)$-neighborhood of $\varphi$, with $\gamma$ as a closed orbit. Thus $\Omega(\psi) \neq \varnothing$, and $\varphi$ is explosive. Q.E.D.

Of course, this argument is a restatement, in the language of quasiorbits, of the perturbation argument at the end of [11]. In particular, this proves the conjecture in [14], that the existence of a strict Liapunov function is equivalent to the nonexistence of $C^{0} \Omega$-explosions (in the completely unstable case).

5. Filtrations for completely unstable flows. A condition for preventing $\Omega$-explosions on a compact space, which is somewhat different in character from those explored so far in this paper, is the existence of a fine sequence of filtrations. This notion was first formulated for diffeomorphisms in [13], and studied for flows (on compact manifolds) in [11]. In the compact case, the nonwandering set is always nonempty, and in fact every orbit tends, in a precise sense, towards this set. Because of this, the idea of a filtration can be reformulated (as it is in [11]) in terms of various kinds of "decompositions" for $\Omega(\varphi)$. However, in the situation of interest to us, $\Omega(\varphi)$ is empty, and so these "decomposition" ideas are somewhat more difficult to formulate. Nonetheless, we can adapt the "filtration" ideas to our situation. One formulation of "filtrations" in a noncompact space is given by Mendes [7]; ours is slightly different.

Definition. A filtration for the flow $\varphi$ is a finite or infinite sequence $\mathscr{T}=\left\{\boldsymbol{M}_{i}\right\}$ of nested, closed submanifolds-with-boundary of codimension zero in $M$, for which $\dot{\varphi}$ is transverse to the boundary of each $M_{i}$, and points inward. More precisely, let $a$ be a negative integer or $-\infty, b$ a positive integer or $+\infty$. Then $\Re$ is a set of submanifolds-with-boundary, $M_{i}$, one for each integer $a<i<b$, such that:

(i) $\operatorname{clos} M_{i} \subset$ int $M_{i+1}$,

(ii) $X=\dot{\varphi}$ is nowhere tangent to any $\partial M_{i}$,

(iii) for $t>0$ and $x \in \operatorname{clos} M_{i}, \varphi(t, x) \in$ int $M_{i}$.

We extend the sequence to $i=a$ and $i=b$ by $M_{a}=\varnothing, M_{b}=M$. We note that in the compact case [11], the sequence is always taken to be finite, while Mendes [7] finds it useful to set $a=0$ and $M_{a}$ nonempty. In Conley's terminology [4], a filtration is a nested collection of isolating blocks with no exit set. The utility of a filtration is that it separates out certain invariant subsets for $\varphi$.

Definition. Given a filtration $\mathscr{N}=\left\{M_{i}\right\}$ for the flow $\varphi$, define the sets

$$
\begin{aligned}
K_{i}(\mathfrak{T}, \varphi) & =\bigcap_{-\infty<t<\infty} \varphi\left(t, \operatorname{clos}\left(M_{i}-M_{i-1}\right)\right), \\
K(\mathfrak{N}, \varphi) & =\bigcup_{a<i<b} K_{i}(\mathfrak{R}, \varphi) .
\end{aligned}
$$


The set $K_{i}$ is the largest invariant subset of $M_{i}-M_{i-1}$; the conditions (ii) and (iii) insure that $K_{i}$ is disjoint from $\partial M_{i} \cup \partial M_{i-1}$, and hence a closed subset of $\operatorname{int}\left[M_{i}-M_{i-1}\right]$. In the compact case, a filtration $\mathscr{N}$ is fine if $K(\Re, \varphi)=\Omega(\varphi)$. In the completely unstable case which interests us, this would mean that $K_{i}=\varnothing$ for each $i$-in other words, that every orbit in $M_{i}-M_{i-1}$ must cross $\partial M_{i} \cup \partial M_{i-1}$ in either forward or backward time. Even in the compact case, it is not always possible to construct a fine filtration. However, it is sometimes possible to construct a sequence of successively "finer" filtrations whose invariant sets converge to $\Omega(\varphi)$.

Definition. 1. A filtration $\mathfrak{N}=\left\{M_{i}\right\}$ refines another filtration $\overline{\mathfrak{T}}=$ $\left\{\bar{M}_{j}\right\}$ if:

(a) for each $i, a<i<b$, there exists $j, \bar{a}<j<\bar{b}$, such that $M_{i}-M_{i-1} \subset$ $\bar{M}_{j}-\bar{M}_{j=1}$.

(b) If $\bar{b}$ (and hence $b$ ) is infinite, then for each $j$ there exists $i$ with $\bar{M}_{j} \subset M_{i}$.

2. A fine sequence of filtrations for $\varphi$ is a sequence $\Re^{\alpha}, \alpha=1,2, \ldots$, of filtrations such that

(a) $\mathfrak{R}^{\alpha+1}$ refines $\Re^{\alpha}$,

(b) $\cap_{\alpha} K\left(\Re^{\alpha}, \varphi\right)=\Omega(\varphi)$.

We can use our earlier results to show that, when $\Omega(\varphi)=\varnothing$, the existence a fine sequence of filtrations is equivalent to the existence of no explosions. The easiest implication to see is necessity of a fine sequence of filtrations for nonexplosiveness:

Lemma 6. If $\varphi$ possesses a strict Liapunov function, then it possesses a fine sequence of filtrations.

Proof. As before, we can assume our given Liapunov function $f$ is smooth and satisfies the stronger condition

$$
X f(x)<0 \text { for all } x \in M .
$$

By composing $f$ with an appropriate $C^{\infty}$ map $R \rightarrow R$ whose derivative never vanishes, we can assume

$$
\sup \{f(x) \mid x \in M\}=1, \quad \inf \{f(x) \mid x \in M\}=0 .
$$

(We note that neither extreme can be achieved, for otherwise $f$ could not continue to decrease along orbits.) We then define a filtration $\mathfrak{T}^{\alpha}=$ $\left\{M_{1}^{\alpha}, M_{2}^{\alpha}, \ldots, M_{2^{\alpha}}^{\alpha}\right\}$ by

$$
M_{i}^{\alpha}=\left\{x \in M \mid f(x)<i / 2^{\alpha}\right\} .
$$

Since every value of $f$ is regular, $\partial M_{i}^{\alpha}=\left\{x \in M \mid f(x)=i / 2^{\alpha}\right\}$ is a hypersurface in $M$, and the condition on $X f$ above insures that $\mathfrak{T}^{\alpha}$ is a filtration for each $\alpha$. It is clear, also, that $\mathfrak{N}^{\alpha+1}$ refines $\mathfrak{T}^{\alpha}$.

Finally, given a point $x \in M$, the fact that $f$ is strict Liapunov insures that 


$$
0<l(x)=\lim _{t \rightarrow-\infty} f\left(\varphi^{t}(x)\right)-\lim _{t \rightarrow \infty} f\left(\varphi^{t}(x)\right) .
$$

But for each point of $K\left(\mathscr{T}^{\alpha}, \varphi\right), l(x)<1 / 2^{\alpha}$. Thus, no point can belong to $\cap{ }_{\alpha} K\left(\mathscr{T}^{\alpha}, \varphi\right)$, and so the sequence is fine. Q.E.D.

Thus, in our main theorem, we have shown that condition 2 (and hence 1, 3 or 4$)$ implies condition 5 . The converse $(5 \Rightarrow 4)$ follows from the following observation:

LEMMA 7. For any filtration, $\Re, R_{C}(\varphi) \subset K(\Re, \varphi)$.

Proof. We will show that $x \notin K(\Re, \varphi)$ implies $x \notin P^{+}(x)$.

Note that, for any $x \notin K(\mathscr{N}, \varphi)$, there exist an index $i=i(x)$ and a time $T=T(x)>0$ such that

(i) $x \in M_{i}-M_{i-1}$,

(ii) $\varphi^{T}(x) \in$ int $M_{i-1}$.

Furthermore, $\varphi\left(1, \operatorname{clos} M_{i-1}\right)$ is a closed subset of int $M_{i-1}$; hence it is possible to pick a function $\varepsilon>0$ such that, for any point $y \in \varphi\left(1, \operatorname{clos} M_{i-1}\right)$,

$$
0<\varepsilon(y)<\frac{1}{2} d\left(y, M-\text { int } M_{i-1}\right) .
$$

We claim that no $(\varepsilon, T+1)$-chain starting from $x$ as above can return to $x$. For, if $x=x_{0}$, then $\varphi\left(T+1+t, x_{0}\right) \in \varphi\left(1, M_{i-1}\right)$, and

$$
\begin{aligned}
d\left(x_{1}, \varphi\left(T+1+t, x_{0}\right)\right) & <\varepsilon(\varphi(T+1+t, x)) \\
& <\frac{1}{2} d\left(\varphi\left(T+1+t, x_{0}\right), M-M_{i-1}\right) .
\end{aligned}
$$

Thus,

$$
\begin{aligned}
d\left(x_{1}, M-M_{i-1}\right)> & d\left(\varphi\left(T+1+t, x_{0}\right), M-M_{i-1}\right) \\
& -d\left(x_{1}, \varphi\left(T+1+t, x_{0}\right)\right)>0
\end{aligned}
$$

and $x_{1} \in M_{i-1}$. Then by induction, $x_{k} \in M_{i-1}$ for all $k>1$. Since $x \in M_{i}-$ $M_{i-1}$, this means no $(\varepsilon, T+1)$-chain starting from $x$ can return to $x$, and so $x \notin P^{+}(x)$. Thus $x \notin K(\mathscr{N}, \varphi)$ implies $x \notin R_{C}(\varphi)$, which is equivalent to the desired result.

We have shown, then, that $R_{C}(\varphi) \subset K(\mathscr{N}, \varphi)$ for any filtration. Thus, for any collection of filtrations, $\mathfrak{T R}^{\alpha}$,

$$
R_{C}(\varphi) \subset \bigcap_{\alpha} K\left(\Re^{\alpha}, \varphi\right) .
$$

In particular, if $\mathfrak{T}^{\alpha}$ are a fine sequence of filtrations for completely unstable $\varphi$, then the right side above, and hence the left side, is empty. Combined with our earlier results, this shows

Proposition 5. If $\Omega(\varphi)=\varnothing$, then $\varphi$ is nonexplosive if and only if it possesses a fine sequence of filtrations.

Although we have established the equivalence of conditions 5 and 1 ; it is 
not clear whether or not, in the completely unstable case, a fine sequence of filtrations can always be replaced by a single, fine filtration. We shall see that such a replacement is not always possible. The example depends on the following observation:

LEMMA 8. If $\Omega(\varphi)=\varnothing$ and $\Re$ is a fine filtration for $\varphi$, then for any $x \in M_{i}$, every orbit in $J_{1}^{+}[x]$ is contained in $M_{i}$ and intersects $M_{i-1}$.

Proof. By condition (ii) in the definition of filtration, $x \in M_{i}$ implies $\varphi(t, x) \in$ int $M_{i}$ for all $t>0$; since $J_{1}^{+}[\varphi(t, x)]=J_{1}^{+}[x]$, we can assume $x \in$ int $M_{i}$.

Suppose $y \in J_{1}^{+}[x]$-that is, suppose $y=\lim \varphi\left(t_{n}, x_{n}\right)$, where $x=\lim x_{n}$ and $\lim t_{n}=\infty$. If $x \in$ int $M_{i}$, we have $x_{n} \in M_{i}$ for sufficiently large $n$. But since $M_{i}$ is positively invariant, this implies $\varphi\left(t_{n}, x_{n}\right) \in M_{i}$, and hence $y \in$ $M_{i}$. Thus, $J_{1}^{+}[x] \subset M_{i}$.

Now suppose some orbit in $J_{1}^{+}[x]$ is disjoint from $M_{i-1}$. We know from the above that it is contained in $M_{i}$; but then we have a nonempty invariant set contained in $M_{i}-M_{i-1}$, contradicting the assumption that $\mathfrak{N}$ is a fine filtration.

CoROllary. If $\Omega(\varphi)=\varnothing$ and $\varphi$ has a fine filtration, then for any pair of points $x, y \in M$, there is a finite upper bound on the integers $n$ for which $y \in\left(J_{1}^{+}\right)^{n}[x]$.

That is, there exists an integer $N=N(x, y) \geqslant 0$ so that any finite sequence of points $m_{1}, \ldots, m_{n-1} \in M$ satisfying

$$
\begin{aligned}
m_{1} \in J_{1}^{+}[x], m_{i+1} & \in J_{1}^{+}\left[m_{i}\right] \text { for } i=1,2, \ldots, n-2, \\
y & \in J_{1}^{+}\left[m_{n-1}\right],
\end{aligned}
$$

has at most $N-1$ elements.

Proof of Corollary. Suppose $\mathfrak{N}$ is a fine filtration with $x \in M_{k}-$ $M_{k-1}$, and assume we have a sequence $\left\{m_{i}\right\}$ as above. Since $m_{1} \in J_{1}^{+}[x]$, the orbit of $m_{1}$ is contained in $M_{k}$, and has nonempty intersection with $M_{k-1}$; by induction, the orbit of $m_{i}$ is contained in $\boldsymbol{M}_{k-i+1}$, and intersects $\boldsymbol{M}_{k-i}$. Thus, we can conclude that $y \in M_{k-n+1}$. On the other hand, there is a unique integer $N$ so that $y \in M_{k-N+1}-M_{k-N}$, and so it follows that $n<N-1$. Q.E.D.

Thus, an obstruction to the existence of a fine filtration would be a pair of points that can be joined by arbitrarily long sequences of prolongational limits as above.

An example of such a situation can be constructed, adapting a few ideas of Mendes [7] and Takens and White [14], as follows: Let $K$ be a Cantor set on the real line. Then the set $M$ of points in the plane defined by

$$
M=\left\{(x, y) \in R^{2} \mid x \notin K \text { or } y>0\right\}
$$


is open, hence a manifold. Take a nonnegative $C^{\infty}$ function $\theta: R^{2} \rightarrow R$ which is zero precisely on

$$
R^{2}-M=\left\{(x, y) \in R^{2} \mid x \in K \text { and } y<0\right\} .
$$

Now consider the flow $\varphi$ on $M$ defined by

$$
\dot{x}=\theta(x, y), \quad \dot{y}=0 .
$$

We note that the complement of $K$ on the $x$-axis is a union of countably many orbits of $\varphi$ (each component of $R-K$ is an orbit). If $x_{1}, x_{2} \notin K$ and $k \in K$ with $x_{1}<k<x_{2}$, then the line segments

$$
L_{n}=\left\{(x, y) \mid x_{1} \leqslant x \leqslant x_{2} \text { and } y=1 / n\right\}
$$

are orbit segments of increasing time-length converging at the ends to $\left(x_{1}, 0\right)$ and $\left(x_{2}, 0\right)$, respectively; so $\left(x_{2}, 0\right) \in J_{1}^{+}\left[\left(x_{1}, 0\right)\right]$. In particular, there exist points in the complement of $K$ which are separated by infinitely many points of $K$. The corresponding points of the $x$-axis can be connected by arbitrarily long "chains" of prolongational limits, and so by the corollary $\varphi$ cannot have a fine filtration. On the other hand, the function $f: M \rightarrow R$ defined by $f(x, y)=-x$ is a strong Liapunov function for $\varphi$, and thus $\varphi$ has a fine sequence of filtrations.

We note in passing that $M$ is diffeomorphic to the plane, $R^{2}$. Of course, it is easy to perturb this situation so as to destroy this phenomenon: for example, make $\dot{y}$ slightly negative everywhere. But it is unclear whether a generic completely unstable flow has a fine filtration.

6. Gradient structure of completely unstable flows. We turn now to the fourth condition of our theorem; the existence of a function and a metric so that $\dot{\varphi}=\operatorname{grad} f$. As we shall see, this condition is merely an elaboration of the existence of a strong Liapunov function.

Proposition 6. If $\Omega(\varphi)=\varnothing$, then $\varphi$ is a gradient flow if, and only if, $\varphi$ possesses a strict Liapunov function.

Proof. If $\varphi$ is gradient, that is, in some metric, $\dot{\varphi}=\operatorname{grad} f$, then clearly $f$ has no critical points, and thus $-f$ is a strict Liapunov function, because

$$
X(-f)=X \cdot \operatorname{grad}(-f)=(\operatorname{grad} f) \cdot(-\operatorname{grad} f)<0 .
$$

On the other hand, suppose that $f$ is a strong Liapunov function for $\varphi$ : $X f<0$ everywhere. Recall that the gradient of a smooth function $f$ depends on the Riemann metric we choose, since it is defined by

$$
d f(v)=v \cdot \operatorname{grad} f .
$$

We will construct a Riemann metric on $M$ so that $X=-\operatorname{grad} f$.

We note that $f$ has no critical points, and so its level sets form a foliation of $M$, of codimension 1 . In particular, the kernel of $d f$ at each point $x \in M$ is a 
hyperplane $H_{x}$ in the tangent space, $T_{x} M$ :

$$
H_{x}=\operatorname{ker} d f(x) \subset T_{x} M .
$$

We know that $X$ is not an element of that hyperplane, so $T_{x} M=H_{x} \oplus V_{x}$ where $V_{x}$ is the subspace of $T_{x} M$ spanned by $X_{x}$. Let $P_{H}$ and $P_{V}$ denote the projections of $T_{x} M$ into these two subspaces.

Now, pick an arbitrary metric, denoting the inner product by $\langle,\rangle_{0}$. Define a new metric by

$$
\langle v, w\rangle_{1}=\left\langle P_{H}(v), P_{H}(w)\right\rangle_{0}+\frac{|X f|}{\langle X, X\rangle_{0}}\left\langle P_{V}(v), P_{V}(w)\right\rangle_{0}
$$

It is easy to check that this new metric is well defined, as smooth as $X$ and $d f$, and that $X_{x}$ is normal to $H_{x}$ at each $x \in M$, with

$$
\|X\|_{1}=|X f|^{1 / 2}=|d f(X)|^{1 / 2} \text {. }
$$

To compute grad $f$, we note that

$$
d f(v)=d f\left[P_{V}(v)+P_{H}(v)\right]=d f\left[P_{V}(v)\right] .
$$

On the one hand, since $V_{x}$ is generated by the vector $X_{x}, P_{V}(v)=\lambda X$ so that $d f(v)=\lambda d f(X)$. On the other hand,

$$
\langle v, X\rangle_{1}=\left\langle P_{V}(v), X\right\rangle_{1}=\langle\lambda X, X\rangle_{1}=\lambda\|X\|_{1}^{2}=\lambda|d f(X)| \text {. }
$$

Noting that $d f(X)<0$, we see that $d f(v)=-\langle v, X\rangle_{1}$ so $X=-\operatorname{grad} f$ as desired.

7. Parallelizability and structural stability. The earlier papers of Mendes [7] and Krych [6] give structural stability theorems for certain classes of diffeomorphisms on open manifolds. In this section, we will examine briefly the relation of these results to our results on completely unstable flows.

Krych [6] studies diffeomorphisms for which $\Omega=\varnothing$ and which, relative to some metric, are isometries. For our purposes, Krych's results can be summarized as follows:

THEOREM (KRYCH). Let $f: M \rightarrow M$ be a diffeomorphism with $\Omega(f)=\varnothing$.

1. There exists a metric on $M$ such that $f$ is an isometry if, and only if, one of the following conditions holds:

(a) For any compact set $C \subset M$, the orbit of $C, \cup f^{n}[C]$, is a closed set.

(b) For every compact set $C \subset M$, there exists an integer $N>0$ such that $f^{n}[C] \cap C=\varnothing$ for $|n|>N$.

2. If $f$ satisfies the conditions of 1 , then it is structurally stable under (strong) $C^{1}$ perturbations.

Mendes [7], on the other hand, studies diffeomorphisms for which $\Omega(f)$ is nonempty. He assumes the following condition:

Definition (Mendes). We say that “ $\infty$ is an $f$-strong attractor (resp. 
repeller) for $x \in M$ " if there exists a neighborhood $V_{x}$ such that, for any compact set $K \subset M$, the intersection $f^{n}[V] \cap K$ is nonempty for only finitely many positive (resp. negative) integers $n$.

He proves several theorems about $\Omega$-stability and structural stability of diffeomorphisms; one assumption common to all of these is that, if $\omega(x)=\varnothing$ (resp. $\alpha(x)=\varnothing$ ), then $\infty$ is an $f$-strong attractor (resp. repeller) for $x$. His structural stability result can be stated as follows:

THEOREM (MENDES). If $f: M \rightarrow M$ is a diffeomorphism satisfying

1. $\Omega(f)$ is discrete,

2. $f$ is Kupka-Smale,

3. $\omega(x)=\varnothing$ implies $\infty$ is a strong $f$-attractor for $x$, and $\alpha(x)=\varnothing$ implies $\infty$ is a strong $f$-repeller for $x$,

then $f$ is structurally stable under $C^{1}$ perturbations.

We shall see that, when $\Omega=\varnothing$, the hypotheses of Mendes and of Krych are equivalent. We shall state the following result simultaneously for cascades (diffeomorphisms) and flows, since the basic arguments and definitions are the same.

Proposition 7. Suppose $\varphi$ is a dynamical system with $\Omega(\varphi)=\varnothing$ on the locally compact manifold $M$. Then the following are equivalent:

(i) (Krych's condition) For every compact set $C \subset M$, the union of $\varphi$-orbits through $C$ is closed.

(ii) (Mendes' condition) $\infty$ is a $\varphi$-strong attractor for every point $x \in M$.

(iii) For every point $x \in M, J_{1}^{+}[x]=\varnothing$.

Proof. (i) $\Rightarrow$ (ii). Take a pair of compact neighborhoods, $U \subset$ int $V$, of $x$, with $V$ (and hence $U$ ) unrevisited. Suppose $\varphi(t, U)$ intersects some compact $K$ for arbitrarily large $t$. Since $V$ is unrevisited, one can pick a sequence of times $t_{n} \rightarrow \infty$ so that $\varphi\left(t_{n}, V\right)$ are disjoint and $\varphi\left(t_{n}, U\right) \cap K \neq \varnothing$. Since the orbit of $U$ is closed, $\cup_{n} \varphi\left(t_{n}, U\right) \cap K$ is compact; but the collection $\varphi\left(t_{n}\right.$, int $\left.V\right)$ is an open cover of this set by infinitely many disjoint open sets, a contradiction.

(ii) $\Rightarrow$ (iii). If $y \in J_{1}^{+}[x]$, let $K$ be a compact neighborhood of $y$ and $V$ a neighborhood eventually disjoint from $K$. This is possible by (ii), but contradicts the definition of $J_{1}^{+}[x]$.

(iii) $\Rightarrow$ (i). If some compact set $C$ has an orbit which is not closed, let $y \in \overline{\operatorname{orbit}[C]}$ - orbit $[C]$. Since any finite part of the orbit of $C$

$$
\bigcup\{\varphi(t, C) \mid a \leqslant t \leqslant b\}
$$

is compact, there must exist points $x_{n} \in C$ and $t_{n} \rightarrow \infty$ with

$$
y=\lim \varphi\left(t_{n}, x_{n}\right) \text {. }
$$


But by compactness of $C$ for a subsequence of the $x_{n}$ 's, there exists $x=\lim x_{n}$ $\in C$. Then $y \in J_{1}^{+}[x]$, contradicting (iii). Q.E.D.

In the case of a flow, the condition (iii) is well known as an equivalent to being parallelizable:

Definition [3]. A flow $\varphi$ on $M$ is parallelizable if there exists a topological space $N$ and a homeomorphism

$$
h: M \rightarrow R \times N, \quad h(x)=\left[h_{1}(x), h_{2}(x)\right]
$$

so that $h(\varphi(t, x))=\left[t+h_{1}(x), h_{2}(x)\right]$ for every $t \in R$ and $x \in M$.

The theory of parallelizability, and its relation to conditions like those above, goes back to Nemytskii and Stepanov [8]. For our purposes, the following theorem suffices; we state it without proof:

TheOREM ([1, Theorem 6] OR [3, p. 49]). A flow $\varphi$ on $M$ is parallelizable if, and only if, for every $x \in M, J_{1}^{+}[x]=\varnothing$.

We see, then, that the analogue of the structural stability theorems of Krych and Mendes, in the case of completely unstable flows, is the following result, which has an easy proof:

THEOREM. A smooth parallelizable flow is structurally stable, under (Whitney) $C^{0}$ perturbations.

Proof. In the case of a smooth parallelizable flow $\varphi$ on a manifold, $M$, one can take $N$ to be a manifold, and $h$ to be a diffeomorphism, in the definition above (this is implicit in Dugundji and Antosiewicz [5, §6]). Then the function $f$, defined by projecting the image of $h$ onto the real factor and multiplying by -1 , is a strict Liapunov function for which we have the especially strong condition

$$
X f=\frac{d}{d t}\left[f \circ \varphi^{t}\right]=-1 .
$$

Note also that $N=h \circ f^{-1}[0]$. Now, if $\psi$ is a flow sufficiently $C^{0}$ near $\varphi$, then the vectorfield $Y=\dot{\psi}$ will satisfy $Y f<-1+\varepsilon$ for some small $\varepsilon>0$. This implies, however, that

$$
\frac{d}{d t} f(\psi(t, x))<-1+\varepsilon
$$

everywhere. Thus, the integral of the above expression for $x \in M$ fixed and $-\infty<t<\infty$ diverges, and hence for every point $x \in M$ there exists a unique time $t=t(x)$ with

$$
f(\psi(t(x), x))=0 .
$$

But then the map $\bar{h}: M \rightarrow R \times N=R \times f^{-1}[0]$ defined by

$$
\bar{h}(x)=(-t(x), \psi(t(x), x))
$$


is easily shown to be a diffeomorphism, and $h^{-1} \circ \bar{h}$ conjugates $\varphi$ with $\psi$. Q.E.D.

Our results on nonexplosiveness can be regarded as necessary and sufficient conditions for $\Omega$-stability of completely unstable flows, and it is natural to ask whether structural stability theorems are equally easy to prove in the case when prolongational limits exist. The answer is "no". An easy example of a nonexplosive flow in $R^{2}$ which is not structurally stable is

$$
\dot{x}=\sin ^{2} x, \quad \dot{y}=\cos x .
$$

the lines $x=n \pi, n=0, \pm 1, \ldots$, are orbits with

$$
((n+1) \pi, y) \in J_{1}^{+}[(n \pi, y)] \text { for all } y,
$$

and so this flow is not parallelizable. On the other hand, for any function $\varepsilon(x)>0$, the flow

$$
\dot{x}=\sin ^{2} x+\varepsilon(x), \quad \dot{y}=\cos x,
$$

is parallelizable, because $\dot{x}>0$ everywhere and $\dot{y}$ is bounded, so that every orbit crosses the $y$-axis exactly once. Since parallelizability is a conjugacy invariant, the two flows are not conjugate, and the original flow is not structurally stable (although it is $\Omega$-stable).

In fact, Takens and White [14] have constructed an open set of completely unstable, nonexplosive flows which are not structurally stable. Structural stability results for completely unstable systems will have to take account of the prolongational limit structure, which can be quite delicate.

\section{REFERENCES}

1. J. Auslander, Generalized recurrence in dynamical systems, Contributions to Differential Equations 3 (1964), 65-74.

2. J. Auslander and P. Seibert, Prolongations and stability in dynamical systems, Ann. Inst. Fourier (Grenoble) 14 (1964), 237-268.

3. N. P. Bhatia and G. P. Szegö, Stability theory of dynamical systems, Springer, New York, 1970.

4. C. Conley, The gradient structure of a flow. I, unpublished IBM Research Report, Yorktown Heights.

5. J. Dugundji and H. A. Antosiewicz, Parallelizable flows and Lyapunov's second method, Ann. of Math. (2) 73 (1961), 543-555.

6. M. Krych, Note on structural stability of open manifolds, Bull. Acad. Polon. Sci. 22 (1974), 1033-1038.

7. P. Mendes, On stability of dynamical systems on open manifolds, J. Differential Equations 16 (1974), 144-167.

8. V. V. Nemytskii and V. V. Stepanov, Qualitative theory of differential equations, Princeton Univ. Press, Princeton, N. J., 1960.

9. Z. Nitecki, Explosions in completely unstable dynamical systems, in Dynamical systems, Proc. Univ. Florida Internat. Sympos., Academic Press, New York, 1977.

10. _ Explosions in completely unstable flows. II. Some examples, Trans. Amer. Math. Soc. 245 (1978), 63-88.

11. Z. Nitecki and M. Shub, Filtrations, decompositions, and explosions, Amer. J. Math. 97 (1976), 1029-1047. 
12. J. Palis, A note on Q-stability, Proc. Sympos. Pure Math., Vol. 14, Amer. Math. Soc., Providence, R. I., 1970, pp. 221-222.

13. M. Shub and S. Smale, Beyond hyperbolicity, Ann. of Math. (2) 96 (1972), 587-591.

14. F. Takens and W. White, Vector fields with no norwwandering points, Amer. J. Math. 98 (1976), 415-425.

15. F. W. Wilson, Smoothing derivatives of functions and applications, Trans. Amer. Math. Soc. 139 (1969), 413-428.

Departigant of Mathibamatics, TUfTs Universtty, Medpord, MassachusetTs 02155 